\title{
CALORIE MEASUREMENT AND CLASSIFICATION OF FRUITS USING IMAGE PROCESSING: A REVIEW
}

\author{
Manpreetkour Basantsingh Sardar \\ PG Research Student, Electronics and Telecommunication Engineering, Maharashtra Institute of Technology, \\ Aurangabad, Maharashtra, India \\ manpreetkoursardar@gmail.com
}

\begin{abstract}
In recent time, the spread of obesity and being overweight has been worldwide significant and is considered as one of the major public health issue. By observing present scenario the researchers have try to resolve them by introducing some methods like calorie measurement system. Therefore by measuring the calories we can keep our health good and fit. Using technology for fruit recognition and estimation of its calories is very useful to spread food culture and awareness among people in the age of obesity due to the bad habits of food consumption and wide range of inappropriate food products. Researchers have been working on developing a reliable image based system for fruit recognition and calories estimation. The system consists of six phases: input fruit photo, photo conversions, photo fragmentation, texture analysis, classification, counting the calorie. Dissimilar approaches have tackled the system from different features. This paper reviews the state of the art of this interesting application.
\end{abstract}

Keywords: Calorie Measurement, Image Segmentation, Feature Extraction, Fruit Recognition.

$* * *$

\section{INTRODUCTION}

In recent era, the spread of obesity and being overweight has been worldwide important and is considered as one of the major public health issue. Similarly, the World Health Organization (WHO) stated that the rate of obesity around the world has surpassed one billion; they predicted that this number might increase to 1.5 billion in 2015. Generally, obesity is defined as the increasing number of fat cells in a person's body [1]. Thus, fatness produces many persistent syndromes such as different types of diabetes, sleep apnea, ischemic stroke, risks of coronary heart diseases, kidney and gall bladder and breast and colon cancer.

Researchers are more interested in obesity cure, and the results show that the lack of balance for energy consumed by human being causes fatness [1]. By keeping above problems in mind some researchers have introduced the calorie balancing system. If we succeed to balance the calories in the human body, it will be useful to control the obesity and overweight. At the basic level the calorie and nutrition imbalance are the result of overweight and obesity. A calorie is a unit of energy that measures how much energy food provides to the body. Body needs calories to function properly [2].
Table -1: Standard Calorie Table

\begin{tabular}{|l|l|l|l|l|}
\hline Fruit Name & $\begin{array}{l}\text { Weight } \\
(\mathrm{mg})\end{array}$ & Calories & $\begin{array}{l}\text { Fat } \\
(\mathrm{g})\end{array}$ & $\begin{array}{l}\text { Protein } \\
(\mathrm{g})\end{array}$ \\
\hline Apple & 100 & 52 & 0.17 & 0.26 \\
\hline Bananas & 100 & 346 & 1.81 & 3.89 \\
\hline Papayas & 100 & 39 & 0.14 & 0.61 \\
\hline Guavas & 100 & 36 & 0.14 & 0.32 \\
\hline Lemons & 100 & 47 & 0.3 & 1.5 \\
\hline Oranges & 100 & 47 & 0.12 & 0.94 \\
\hline Watermelon & 100 & 30 & 0.14 & 0.56 \\
\hline
\end{tabular}

Table 1 demonstrates that the weight of a fruit and its calories referred as a standard database [3]. Every fruit have its own calories proportional to weight. By referring to the above table calories can be calculated.

\section{TRADITIONAL APPROACHES}

The Doubly Labeled Water (DLW) method was developed in the early 1950s by Lifson and McClintock in 1966 [4]. DLW was widely used for a long time in measurement issues related to dietary intake studies to estimate energy expenditure. Biro, G. et.al developed twenty four hour dietary recall method is basically means an interview. In this method the trainer request to user or patient to memorize the details of fruit consumption and maintain the records of individual person on paper at every twenty four hours [5].

Two objectives are behind the usage of the dietary intake assessment, which are figuring if any society's population is taking enough nutrients like crabs, proteins, sodium or 
calcium or discovering if any person is taking more than he or she needs [6]. Generally, the dietary assessment recall causes an underestimation of energy intake [7]. In addition, Schoeller. D.A. explains the incidence of underreporting is too high due to data collection methods [8]. The main advantage of the 24-hour dietary recall is that a professional interviewer will complete the food list, but this method is expensive. Clinical approach gives the correct record of food consumption week to week [9].

The difficulty with manual approach is that citizens or patients are not able to remember what they ate, improper guidance, unable to memorize and record of food intake and do not get expert advice. Mingui Sun et.al proposes a new method based on card [10]. While capturing the food image calibration card has to be placed next to the food. In case if the card was misplaced or person won't bring along then this system fails. J. Beasley proposes a system based on smart digital device known as Personal Digital Assistive (PDA) for calorie estimation purpose [11]. L. E. Burke et.al proposed the system which deals with the user to record their food intake on cell phone, but study proved that it is time consuming method and give calorie estimation error [12].

Table -2: Traditional Methods Summary Table

\begin{tabular}{|l|l|l|}
\hline Methods & Description & Limitations \\
\hline DLW & Give H2O and check the urine samples. & Time consuming \\
\hline 24HRS & Interview & Expert dietitian required \\
\hline Clinical & Maintaining the records & People not remembering exactly what they ate \\
\hline Calibration card & Uses reference cards & Hard to maintain cards \\
\hline PDA & Take info via phone & Take long time \\
\hline
\end{tabular}

\section{MODERN APPROACH}

As a substitute for the old dietary assessment methods, researchers developed electronic devices for measuring food intake connected to computers. Accordingly, the usage of smart technological devices in the food calorie measurement and estimation method became famous in health care for data collection and easy to handle.

\subsection{Electronics Dietary Assessment Approaches}

Academic journal have been used to measure several categories, such as physical activities, sleep, pain, heart rate, medication taken, and food intake and energy expenditure [12]. Thus, much food intake measuring software has been developed such as Calorie Counter, Meal Snap and Veggie Vision. Amft. O. et.al developed an Automatic Dietary Monitoring (ADM) system based on weight of individual bites taken. ADM system uses sensors fix on human body to monitor weight of person's bites of food. The sensor is sound sensor which records the sound of chewing continuously [13].

Specifically, system received the intake signals via a wristworn acceleration sensor, a microphone in the external ear canal to record chewing sounds (This area provides a loud chewing signal without environmental sounds), and an electromyography (EMG) sensor at the throat to measure swallows. K. Chang et.al developed an embedded system based on radio frequency and surface sensors. They embedded the dining table with surface sensor [14]. The Radio Frequency Identification (RFID) as a surface sensor to check the type of food eaten and measuring scale on dining table can be used to measure the food weight. But there are many problems associated to this system, include the use of system, space requirement and the difficulty of the RFID tag setup.

\subsection{Image Processing Based Approach}

The system is based on image processing having different phases and each phase has different approaches and is depend on each other, the overall aggregation of the phases is reflected in the performance of the system. The Figure 1 shows the general phases of the system.

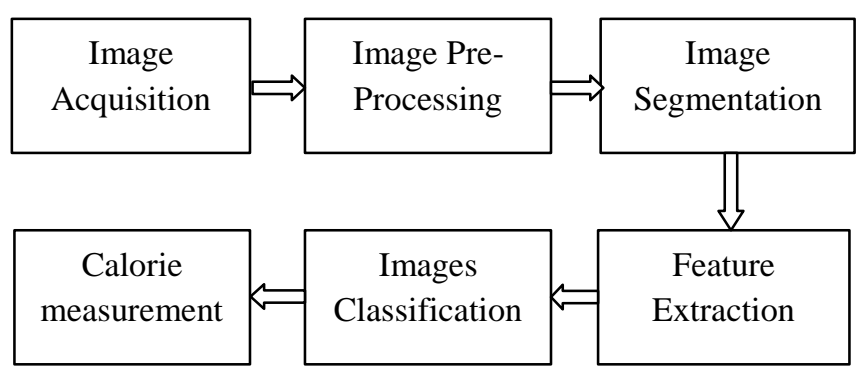

Figure 1: General Block Diagram

\subsubsection{Image Acquisition}

Pouladzadeh. $\mathrm{P}$ et.al have required the thumb of the user while clicking the photo of food in order to be used as a reference [15]. Martin. C. K. et.al approach was based on calibration card used as reference card in the pictures [16]. The input to the pattern recognition system is usually transducer, such as a camera or a microphone array [17].

In mostly all the fruit sorting machines developed so far this is a camera, to capture the color images of the fruits as an input to the pattern recognition system [18, 19]. A Charge Coupled Device (CCD) camera mount on the top of the imaging box transmits images to the computer attached of size $480 \times 640$ pixel in JPEG format [20]. 


\subsubsection{Image Pre-Processing}

By using the mobile camera or high definition camera the photos of fruits are captured, but it cannot be used directly because it has noise due to dust and light effect. Hence, to reduce the noise and blur the primary step is image preprocessing. Researchers used different types of filters, image cropping, resizing, Thresholding, morphological operations. Neelamegam $P$. et.al have been proposed the blurring removal techniques [21]. Chandra Sekhar Nandi et.al proposed intensity adjustment by scaling the input image to get the desired size, minor lighting problems, and then performed adaptive equalization of the color histogram finally performed a linearly averaging kernel to remove noise and blur [20]. Sun, D.W, \& Du, C.J have been developed a system which remove light reflections and shadows [22].

\subsubsection{Image Segmentation}

Segmentation of an image separates the image from its background area. Stick Growing and Merging (SGM) approach was used by Sun, D.W and Du, developed an algorithm for food images that get segmented into different sub regions [22]. The algorithm consists of three steps: gray value images computation from RGB color components, estimation of global threshold, and morphological operation [23]. Shervin and Abdulsalam proposed the three segmentation methods based on contour, color, and texture [24].

\section{Color Features:}

Finding the contour of objects the graph cut method is useful. The Gabor filters are used for texture segmentation which measures the local texture properties in the frequency domain [25]. Pyramidal mean-shift filtering, a region growing algorithm and region merging have been used for image segmentation [26], this method was evaluated only on carbohydrate food type with plate and keeping same color in the background. He. Y. et.al have performed comparative study between three states of the ability methods of image segmentation in the food image application study [27]. Three methods of segmentation on food images: active contour, normalized cuts, and local variation. Their study showed that local variation was more consistent in terms of performance with changing the configuration parameters comparing with the other two methods. Also, active contour has a performance similar to local variation as the number of initial contour increases, with a drawback of overhead of computational complexity. From this study, it can be seen that initial information provided from prior knowledge such as the number of segments are an essential part of the functionality of the three methods. Typically, in segmentation methods there is a concern about the computational time of the execution.

\subsubsection{Feature Extraction}

Mostly in case of color images the average Red, Blue and Green color values, total number of pixels, total of boundary pixels, length of major and minor axis etc makes feature space for classification [18, 19, 20, 28, 29]. Mango sorting have used 27 features [20], Date classification have used 15 features [19]. We can summarize all features one by one.

Table -3: Contrast of Color Descriptors

\begin{tabular}{|l|l|l|}
\hline Methods & Strengths & Weaknesses \\
\hline Color histogram [30] & Simple to compute & Dimensional, noisy \\
\hline Correlogram [31] & Easy to use, frequency info & costly, noise sensitive, revolution \\
\hline CM [32] & compressed, tough & Unable to describe all color parameters, no frequency components \\
\hline SCD [33] & Scalability & No special information \\
\hline CSD [33] & Spatial information & Noise sensitive, rotational \\
\hline CCV [34] & Frequency domain & High dimension \& computational cost \\
\hline
\end{tabular}

Texture features:

Table -4: Contrast of Texture Descriptors

\begin{tabular}{|l|l|l|}
\hline Methods & Strengths & Weaknesses \\
\hline Spatial texture [35] & $\begin{array}{l}\text { Easy to understand, without losing info extract any } \\
\text { shape info }\end{array}$ & Noisy system and produces distorted output \\
\hline Spectral texture [36] & Robust, need less computation & Need square image regions with sufficient size \\
\hline
\end{tabular}

\subsubsection{Images Classification}

For classification, Support Vector Machine (SVM) was the most used type of classifier among all different types of classifiers [37, 38]. Maruyama .Y et.al have used Bayesian probabilistic for classification [38]. Others have used Radial Basis Function (RBF) kernel for classification, some approaches have used feed forward Neural Network (NN). Sachin et.al proposed the jatropha classification system based on fuzzy logic [29]. Date fruit classifies have tested almost all techniques like K-means, Linear Discriminate Analysis, Neural Networks; it was found that neural networks give the best results and accuracy up to $99 \%$ [19]. 


\subsubsection{Calorie Measurement}

This is final phase of the system. Some researchers used simple look-up table approach, while some used based on different features predetermined algorithms, some used linear regression, mathematical approach.

\section{CONCLUSIONS}

Based on the earlier literature review, it is clear that all approaches follow a systematic framework that consists of pre-processing and post-processing approach. According to fundamental nature, procedure of image based measurement system resides in the part of accurate object classification. Actually, there is no entirety agreement on the most efficient type of features or classifier to be used in the fruit recognition part. But good and accurate fruit recognition requires accurate fruit segmentation. Therefore, the most essential and challenging aspect of this application is fruit segmentation, recognition and classification. Unfortunately, the non-well predefined shape and characteristic of fruit causes add hurdles that researchers are trying to overcome. Researchers have tried to overcome the difficulty of object segmentation by adding an interactive approach to the scheme, while others have just avoided this challenge by simplifying the testing scenarios to very distinctive and well-defined food objects scenario. Overcoming these problems researcher faced some issues such as accuracy, time utilization and cost effective system. Challenge to the new investigators is improve the existing systems with optimized software and ease of use of the application developed. By using image processing in MATLAB software it is easy to construct system with accuracy and cost effectiveness.

\section{ACKNOWLEDGEMENT}

This project review is partially supported by Dr.Sayyad Ajij, Associate Professor, MIT College \& thankful for his guidance and support.

\section{REFERENCES}

[1]. Bray, George A., \& Bouchard, Claude, "Handbook of Obesity", $2^{\text {nd }}$ ed., Marcel Dekker, New York, USA, 2004.

[2]. Kim E. Barrett, Scott Boitano, Susan M. Barman and Heddwen L. Brooks, "Digestion, absorption and nutritional principles", in Ganong's review of medical physiology, USA, ch.27, 2009.

[3]. Health Canada Nutrient Values, 2011

[4]. Shetty. P, "Human energy requirements: where are we now? Issues emerging from the 2001 expert consultation on energy in human nutrition", Food, Nutrition, and Agriculture, vol. 30, pp. 5-13, 2002.

[5]. Biro, G., Hulshof, K., Ovesen, L., \& Cruz, "J.A. Selection of methodology to assess food intake", European Journal of Clinical Nutrition, pp.25-32, 2002.

[6]. Garrow.J, "Human nutritionist's guilty secret", BNF Nutrition Bulletin, pp.103, 1995.
[7]. Hambrus, L., "Dietary assessments: How to validate primary data before conclusions can be drawn", Scandinavian Journal of Nutritional Naringrs forskning, 142:66, 1998.

[8]. Schoeller, D.A., "How accurate is self-reported energy intake?" Nutrients Review 48:373-9, 1990.

[9]. R. Almaghrabi, G. Villalobos, P. Pouladzadeh, and S. Shirmohammadi, "A Novel Method for Measuring Nutrition Intake Based on Food Image", in Proc. IEEE International Instrumentation and Measurement Technology Conference, Graz, Austria, pp. 366 - 370, 2012.

[10].Mingui Sun et al., "Determination of food portion size by image processing", Engineering in Medicine and Biology Society, pp. 871 - 874, 2008.

[11].J. Beasley, "The pros and cons of using PDA's for dietary self monitoring", Diet Assoc, vol. 107, no. 5, pp.739, 2007.

[12].L. E. Burke et al., "Self-monitoring dietary intake: current and future practices", Journal of renal nutrition the official journal of the Council on Renal Nutrition of the National Kidney Foundation, vol. 15, no. 3, pp. 281-290, 2005.

[13].Amft, O., Kusserow, M., \& Tröster, G., "Bite weight prediction from acoustic recognition of chewing", IEEE Transactions on Biomedical Engineering, 2009.

[14].K. Chang et.al, "The diet-aware dining table: Observing dietary behaviors over a tabletop surface," Proceedings of Pervasive Computing- 4th International Conference, pp. 366-382, 2006.

[15].Pouladzadeh, P., Shirmohammadi, S., \& Al-Maghrabi, R., "Measuring calorie and nutrition from food image", In IEEE, Transactions on Instrumentation and Measurement, 2014.

[16].Martin, C. K., Kaya, S., \& Gunturk, B. K., "Quantification of food intake using food image analysis", In Annual International Conference of the IEEE Engineering in Medicine and Biology Society, pp.6869-6872, 2009.

[17].Richard O. Duda, Peter E. Hart, David G. Stork, "Pattern Classification", Second Edition, Wiley publications, 2007.

[18].Youssouf Chherawala, Richard Lepage and Gilles Doyon, "Food Grading/Sorting Based on Color Appearance trough Machine Vision: the Case of Fresh Cranberries", IEEE Conference publications, ICTTA, 2006.

[19]. Abdulhamid Haidar, Haiwei Dong and Nikolaos Mavridis, "Image Based Date Fruit Classification", IEEE Conference on Ultra modern Telecommunications and Systems, pp. 369 - 375, 2012.

[20]. Chandra Sekhar Nandi, Bipan Tudu, and Chiranjib Koley, "A Machine Vision-Based Maturity Prediction System for Sorting of Harvested Mangoes", in IEEE Transactions on Instrumentation and measurement, vol. 63, no. 7, p.p.1722-1730, 2014.

[21].Neelamegam, P., Abirami, S., Vishnu Priya, K., \& Rubalya Valantina, S., "Analysis of rice granules using image processing and neural network", In IEEE 
Conference on Information \& Communication Technologies (ICT), 879-884, 2013.

[22].Sun, D.-W., \& Du, C.-J, "Segmentation of complex food images by stick growing and merging algorithm", Journal of Food Engineering, 2004.

[23]. Domingo mery, Fransco pedreschi, "Segmentation of colour food images using a robust algorithm", Journal of Food Engineering.

[24]. Shervin shirmohammadi and abdulsalam yassine, "Using Graph cut segmentation for food calorie measurement", conference paper, June 2014

[25].k. jain and F. Farrokhnia, "Unsupervised texture Segmentation using Gabor filters", Pattern Recognition, vol.24, pp. 1167-1186, 1991.

[26].Anthimopoulos, M., Dehais, J., Diem, P., \& Mougiakakou, S., "Segmentation and recognition of multi-food meal images for carbohydrate counting", In IEEE 13th International Conference on Bioinformatics and Bioengineering, 2013.

[27].He, Y., Khanna, N., Boushey, C. J., \& Delp, E. J., "Image segmentation for image-based dietary assessment: A comparative study", In 2013 International Symposium on Signals, Circuits and Systems (ISSCS), IEEE, 2013.

[28]. Youssouf Chherawala, Richard Lepage, Gilles Doyon, "Food Grading/Sorting Based on Color Appearance trough Machine Vision: the Case of Fresh Cranberries", IEEE, 2006.

[29].Sachin Syal, Tanvi Mehta, \& Priya Darshni, “ Design \& Development of Intelligent System for Grading of Jatropha Fruit by Its Feature Value Extraction Using Fuzzy Logics", In International Journal of Advanced Research in Computer Science and Software Engineering, vol. 3, No. 7, 2013

[30].K. Jain and A. Vailaya, "Image retrieval using colour and shape", Pattern Recognition, vol. 29, no. 8,pp. 1233-1244, 1996.

[31].J. Huang, S. Kuamr, M. Mitra, et al., "Image indexing using colour Correlogram", In Proc. CVPR, pp. 762765, 1997.

[32].M. Flickner, H. Sawhney, W. Niblack, et al., "Query by image and video content: the QBIC system", IEEE Computer, vol. 28, no. 9, pp. 23-32, 1995.

[33].D. S. Zhang, Md. M. Islam and G. J. Lu, "A review on automatic image annotation techniques", Pattern Recognition, vol. 45, no. 1, pp. 346-362, 2012.

[34].G. Pass and R. Zabith, "Histogram refinement for content-based image retrieval", In Proc. Workshop on Applications of Computer Vision, pp. 96-102,1996.

[35].B. S. Manjunath and W. Y. Ma, "Texture features for browsing and retrieval of large image data", IEEE PAMI, vol. 18, no. 8, pp. 837-842, 1996.

[36].S. E. Grigorescu, N. Petkov and P. Kruizinga, "Comparison of texture features based on Gabor filters", IEEE TIP, vol. 11, no. 10, pp. 1160-1167, 2002.

[37].Pouladzadeh, P., Shirmohammadi, S., \& Arici, T., "Intelligent SVM based food intake measurement system", In IEEE International Conference on Computational Intelligence and Virtual Environments for Measurement Systems and Applications (CIVEMSA), pp.87-92, 2013.

[38].Maruyama, Y., de Silva, G. C., Yamasaki, T., \& Aizawa, K., "Personalization of food image analysis", In 16th International Conference on Virtual Systems and Multimedia (VSMM), pp.75-78, 2010. 